Portland State University

PDXScholar

2016

\title{
Determining the Relationship between White Matter Volume and Processing Speed in Adolescence
}

Julius Laxamana

Portland State University

Follow this and additional works at: https://pdxscholar.library.pdx.edu/honorstheses

Let us know how access to this document benefits you.

\section{Recommended Citation}

Laxamana, Julius, "Determining the Relationship between White Matter Volume and Processing Speed in Adolescence" (2016). University Honors Theses. Paper 315.

https://doi.org/10.15760/honors.273

This Thesis is brought to you for free and open access. It has been accepted for inclusion in University Honors Theses by an authorized administrator of PDXScholar. Please contact us if we can make this document more accessible: pdxscholar@pdx.edu. 


\title{
Determining the Relationship between White Matter Volume and Processing Speed in Adolescence \\ By
}

\author{
Julius Laxamana
}

An undergraduate honors thesis submitted in partial fulfillment of the

\author{
requirements for the degree of \\ Bachelor of Science \\ in \\ University Honors \\ and \\ Psychology
}

Thesis Adviser:

Bonnie Nagel, Ph.D

Portland State University 


\section{ABSTRACT}

Neuroimaging techniques have recently been used to research and identify relationships between structure and function. Because adolescence is a critical period for development of the brain, the present study's goal was to identify the possible relationships between white matter (WM) volume and processing speed during this period. White matter was of particular interest as it consists of the myelinated axons of some nerve cells which are essential for nervous system functioning. The WM volume of 157 adolescents (ages 12-17) were determined from T1weighted images via FMRIB's Automated Segmentation Tool (FAST), whereby the brain was separated into WM, gray matter (GM), and cerebral spinal fluid (CSF). A composite score for processing speed was indicated by a z-normalized mean score from the Wechsler Abbreviated Scale of Intelligence (WASI) block design test, Paced Auditory Serial Addition Test (PASAT), Delis-Kaplan Executive Function System (D-KEFS) which included raw scores of Color-Word Conditions 1-4, verbal and spatial working memory 2-back tasks, and the hit reaction time score from the Continuous Performance Test (CPT). Results suggested that there was a small positive relationship between WM volume and age at the time of the scan, which agrees with several reported literatures. Processing speed was defined as a more negative value below the mean; the initial hypothesis was further supported as a negative relationship between WM volume and age to processing speed in adolescence observed. Although the correlation and significance was relatively low between the variables, the sample suggests that as an adolescent's WM volume or age increased or decreased, processing speed was reflected as marginally quicker or slower.

\section{INTRODUCTION AND BACKGROUND}

The human brain is primarily composed of grey matter (GM) and white matter (WM). GM consists of neuronal cell bodies, neuropil, glial cells, synapses, and capillaries, while WM is primarily composed of glial cells and myelinated axons. The GM's color represents the neuronal cell bodies, while WM's color is due to the myelin sheath. As an insulator on certain axons, myelin increases the speed of signal impulses via saltatory conduction. WM functions to connect areas of GM and sends these signals to a variety of regions including the brainstem and limbic system [1]. These signals are used as the nervous system's communication, and can be translated into cognitive functioning processes or motor processes $[1,7]$. The speed of the signal transmitted is related to the thickness and degree of myelination, and damage to aforementioned regions results in a reduction in functional processing speed [30]. Diseases that damage WM or myelin, such as Alzheimer's, multiple sclerosis (MS), or other factors like lesions also result in reduced function or ability to send these signals $[6,10,32]$.

In the developmental period of adolescence, both GM and WM undergo varying structural maturation [16, 18, 23, 31]. However, increases in GM volumes peak well before an individual's 20's, whereas increases in WM volume peaks in middle age $[18,31]$. In general, WM has been determined to increase significantly during adolescence $[1,15,18,21,25,26,28]$ yet decreases in later adolescent stages [13,15,26,29]. There is also evidence to suggest that WM develops differently between males and females; men have a parallel rate of growth in both GM and WM to cranial volume, whereas women increase in WM at a slower rate in relation to cranial volume $[3,9,11,13,35]$. Furthermore, after accounting for total intracranial volume development, men tend to have a peak higher percentage of WM whereas women have a higher percentage of GM [18]. Several studies have reported sex-specific developmental differences in the relations between cerebral structure and function [35], yet conversely some have observed no significant interactions of sex and cognitive measures when age was not included [29]. However, 
many of these studies have failed to find these relationships in large sample sizes whereby $(\mathrm{N}>100)$.

The use of imaging techniques to identify structural components and functionality of the brain is still a new field of research. There are three types of neuro-imaging techniques that have been frequently used in order to assess the suspected relationship between WM and processing speed: Magnetic resonance imaging (MRI), diffusion tensor imaging (DTI), and functional magnetic resonance imaging (fMRI). MRI is a technique that began to see heavy diagnostic usage within the last two decades. With the ability to determine clear structural brain images, MRI scans have opened up a wide range of diagnostic and research opportunities which have included mapping anatomical regions [5]. The relationship between structure and function has been reported through methods such as identifying physical damages to regions and their respective effects, or in some cases the gradual growth or deterioration [14]. For the purposes of this thesis, MRI was singularly used.

DTI is a technique that records fractional anisotropy (FA) -- or the diffusion of water molecules along the main direction of axon bundles and their myelin. This water diffusion is directionally predisposed to following the axon bundles, which reveals the microstructural components of the WM. Structural images of the WM tracts along targeted areas of the brain can be visually recorded in both 2 and 3-dimensional images. Although a powerful tool of WM visualization, DTI suffers from the drawbacks of frequent artifacts, partial volume mapping, and the inability to cope with non-Gaussian diffusion [2]. DTI is not used for total volumetric determination and is therefore commonly coupled with preexisting MRI-determined WM volumes, whereby there have been reports of protracted WM development over adolescence. [1]. Some studies have used DTI to assess how physical damages such as lesions affect the liquid diffusion, as well as WM maturation over periods such as adolescence [1]. It has been reported in some cases that damage to white matter tracts may underlie age-related cognitive decline [10, 34] Nevertheless, many of these studies have used a sample of either elderly or adult participants in determining processing speed [26,34]; in the cases of children and adolescents included, the sample remained relatively small $(\mathrm{N}<100)$.

The present study looks to build upon the suggested relationships between brain matter structure and function, and therefore the identification of FA through DTI and fMRI in adolescence are particularly relevant. fMRI is frequently coupled with DTI or MRI in order to observe a possible relationship between different brain regions and their activity. In one study, fMRI and DTI was used to test the hypothesis that white matter integrity, as indexed from the FA, was a mechanism of slowing in episodic memory retrieval $[6,14,34]$. Although positive correlations between maturation and increased brain activity have been reported, cases of fMRI studies had a sample of participants primarily above the age of adolescence; these were in periods of slowing WM growth that is conversely reported in younger counterparts $[4,11,27$ 29]. However, there have been several studies on WM structure in relation to processing speed within young participants as well -- there are some reports of reduced FA observed in children with autism [4]. Differential effects of regional variations in diffusion properties on memory and functional speed have also been reported, as well as some effects on processing speed with regards to physical damages like lesions on WM tracts $[8,10,32]$. These studies suggest that, among other factors, the structure of WM is significant in relationship to the functionality of the brain and its processes. The present study looks to identify the possible extent to which the amount of the structure - volume in this case - may relate to the processing speed of adolescence in a larger sample. Although it has been suggested that damage and inhibition of structures has 
adverse effects, conversely how might a higher amount of volume contribute to cognitive speed? Much of the research in the above cases using DTI and fMRI depended heavily on the structural WM determination from MRI [4, 31, 32]. A large sample size and varying participant ages was a limiting factor; the goal of the present study was to report on a large sample of only adolescents (ages 11-17 at scan date used) WM volume. Based on previous evidence [6, 25, 28, 32], mature WM tracts have been reported to greater processing speed. Therefore, it is hypothesized that in this developmental period, processing speed will be positively related to a great amount of WM as most likely related by an older age. It is also expected that a negative relationship be found with lower volumes of WM -- immature, younger ages -- and processing speed.

\section{METHODS}

\section{Participants}

The data used was provided via Oregon Health and Science University's (OHSU) Developmental Brain Imaging Lab in Portland, OR. The present sample consisted of 157 healthy adolescent participants (ages 12-17 of scan date used) that primarily consisted of at-risk youth adolescents from the local community. 82 males and 75 females were examined, of which were selected via participation of several pre-determined measures of processing speed. The sample was taken by screening the parent data set for only those who completed the tasks listed above. The parent study from which the sample was taken was part of an ongoing study regarding atrisk youth, whereby participants were selected via exclusionary criteria that included lefthandedness [22], DSM-IV psychiatric diagnoses, absence of family history information, serious medical problems, significant head trauma, mental retardation or learning disabilities, psychotic illness in a biological parent, prenatal exposure to drugs or alcohol, and MRI contraindications. The study from which the data was collected was approved by the Oregon Health \& Science University (OHSU) Institutional Review Board.

\section{Definition of Processing Speed}

In order to determine a definition for processing speed in the present study, raw scores from multiple tests were used. Specifically, the block design test from the Wechsler Abbreviated Scale of Intelligence (WASI), Paced Auditory Serial Addition Test (PASAT), Color-Word Conditions 1-4 subtests from the Delis-Kaplan Executive Function System (D-KEFS), verbal and spatial working memory 2-back tasks, and the hit reaction time score from the continuous performance task (CPT). All raw scores were then z-normalized in the database, whereby higher scores reflected slower reaction times. The mean of the z-normalized score was taken for each individual, and a multiple linear step-wise regression was used with age as the first step and processing speed as the second. All statistical analyses were performed in SPSS (SPSS for Windows, release 17.0, 2008; SPSS Inc., Chicago, IL).

\section{Image Acquisition}

Participants were scanned at OHSU's Advanced Imaging Research Center on a 3.0-Tesla Siemens Magnetom Tim Trio (Siemens Medical Solutions, Erlangen, Germany). Anatomical, high-resolution T1-weighted MPRAGE structural scans were collected in the sagittal plane (Time repetitions $[\mathrm{TR}]=2,300 \mathrm{~ms}$, time to echo $[\mathrm{TE}]=3.58 \mathrm{~ms}$, inversion time $[\mathrm{TI}]=900 \mathrm{~ms}$, flip angle $=10^{\circ}, \mathrm{FOV}=240 \times 256 \mathrm{~mm}$, voxel size $=1 \times 1 \times 1.1 \mathrm{~mm}, 160$ slices, acquisition time $=9: 14)$. Functional T2-weighted images were also taken of a majority of the participants in the 
parent study; however, the present study utilized only the T1-weighted MPRAGE structural scans collected in the sagittal plane.

\section{Image Processing}

Analysis of Functional NeuroImages (AFNI) was used to reconstruct the raw Digital Imaging and Communications in Medicine (DICOM) files to make a MPRAGE [20]. Functional masks through AFNI were previously created by the parent study; in the present study each individual's mask was simply checked for any errors of or outside the skull that may have been incorrectly marked as target tissue.

\section{Segmentation and WM Volume Determination}

FAST (FMRIB's Automated Segmentation Tool) was used to segment the 3D T1-weighted images of the brain [36]. The images were segmented into three tissue types: GM, WM, and CSF. The command protocol for segmentation was as follows:

- Creation of a new directory for the segmented brain

○ mkdir FAST

- Creation of a skull-stripped brain in the new directory

○ cp [subject]-anat-stripped+orig* FAST

○ cd FAST

- Conversion of the .BRIK and .HEAD files to .nii files

○ 3dAFNItoNIFTI [subject]-anat-stripped+orig

- FAST GUI

○ Fast

- Number of input channels: 1

$\circ$ Input image: click the folder and find [subject]-anat-stripped.nii

- Image type: T1-weighted

- Output image basename: the same basename as the input image

- Number of classes: 3 (GM, WM, CSF)

○ Output images: "Binary segmentation: also output one image per class" and "partial volume maps" are checked

○ Go

- Creation of a copy of the white matter file with a new name

○ cp [subject]-anat-stripped_seg_2.nii.gz ./[subject]-anatstripped_WM_JL.nii.gz

- Edit of AFNI Mask

o afni

- Determination of voxel number included in edited mask

○ 3dBrickStat -count -non-zero [subject]-anat-stripped_WM_JL.nii.gz

*where JL was used as a name for the file. 
The final output from FAST was an approximately six-digit number indicating the number of WM voxels detected. This number was then multiplied by the dimensions of a voxel in the T-1 Weighted-Image, or $1 \times 1 \times 1.1 \mathrm{~mm}$ to determine the WM volume.

\section{RESULTS}

A multiple linear regression and step-wise method was used to analyze the data. WM volume was used as the dependent variable while age was input at the first step and the composite processing speed score on the second step. The following tables were generated through SPSS below; mean z-scores again had more negative values reflecting a faster processing speed. Therefore, a value closer to 0 represented the mean or normal processing speed of the individual in comparison to the sample, and a more positive value represented slower processing speeds (or a farther deviation away from the sample mean).

Table 1: General descriptive statistics of multiple linear regression step-wise calculation with the sample of 157 adolescents.

\section{Descriptive Statistics}

\begin{tabular}{|l|r|r|r|}
\hline & \multicolumn{1}{|c|}{ Mean } & Std. Deviation & \multicolumn{1}{c|}{$\mathrm{N}$} \\
\hline WM_Volume & 563827.4331 & 55744.02509 & 157 \\
Age_At_Scan & 14.4393 & 1.39628 & 157 \\
Mean_Z_Score & -.0045 & .41203 & 157 \\
\hline
\end{tabular}

Table 2: Pearson Correlations and step-wise significance of WM volume influenced by age and composite processing speed z-score. It was determined that the age of the scan had a positive correlation value of approximately 0.010 while the mean $z$-score had a negative correlation to WM volume by -0.070 and -0.281 to the age of the individual.

\begin{tabular}{|ll|r|r|r|}
\hline \multicolumn{1}{|c|}{ Correlations } \\
\hline Pearson Correlation & WM_Volume & Age_At_Scan & Mean_Z_Score \\
& WM_Volume & 1.000 & .010 & -.070 \\
& Age_At_Scan & .010 & 1.000 & -.281 \\
& Mean_Z_Score & -.070 & -.281 & 1.000 \\
\hline Sig. (1-tailed) & WM_Volume &. & .451 & .193 \\
& Age_At_Scan & .451 &. & .000 \\
& Mean_Z_Score & .193 & .000 & \\
\hline N & WM_Volume & 157 & 157 & 157 \\
& Age_At_Scan & 157 & 157 & 157 \\
& Mean_Z_Score & 157 & 157 & 157 \\
\hline
\end{tabular}

It was determined that there was a small positive correlation (0.010) between WM volume and the age of the individual at the time of the scan. However, the processing speed z-score represented a negative correlation with both WM volume and the age of the scan. This data suggests support for the initial hypothesis; the positive relationship between WM volume and 
age represents that as one increases or decreases, the other will tend to do the same. This could be generalized to the suggestion that as an individual or adolescent gets older, the WM volume also increases - which is a generalization that has been supported by previous research within this paper. Furthermore, the table represents a negative correlation between the composite processing speed z-score and both the dependent variable of WM volume and co-independent variable of age. From this sample analysis it is suggested then that as both WM volume and age increases in value for an individual, the processing speed will inversely drop farther below the mean z-score. This also supports the present studies' initial hypothesis because the more negative $\mathrm{Z}$-scores are representative of faster processing speeds as determined by the measures listed. A positive relationship between the processing speed $\mathrm{z}$-score with WM volume and age would then suggest that as adolescents in the sample got older, their speed would deviate farther and slower above the mean. However, the analysis emphasizes that the relationship - in either positive or negative case - was relatively small. Although the analysis of the sample was in agreement with in initial hypotheses, the significance was not particularly high with values remaining below 0.5.

For the sake of extending the analysis and to observe multiple data characteristics, another regression was observed without the step-wise method. The independent and dependent variables were input and entered through SPSS without inputting a specific method. Therefore, the following tables represent the two independent variables of age and mean z-score forced into the multiple regression equation, irrespective of their determined statistical significance. Through this second analytical observation it was determined that the correlational table (table 2) did not change, yet there was significant differences found in the ANOVA. The significance of 0.681 was much higher in this case as expected without accounting for the step-wise independent variables.

Table 3: ANOVA run through enter method of multiple linear regression of SPSS, whereby independent variables were used in the regression equation regardless of statistical significance. The significance level is relatively high in comparison to the correlations found in Table 2.

ANOVA $^{a}$

\begin{tabular}{|c|c|c|c|c|c|c|}
\hline \multicolumn{2}{|c|}{ Model } & \multirow{2}{*}{$\begin{array}{r}\text { Sum of Squares } \\
2410564747.01 \\
5\end{array}$} & \multirow{2}{*}{$\begin{array}{ll}\text { df } & \\
& \\
& \end{array}$} & $\frac{\text { Mean Square }}{1205282373.50}$ & $\begin{array}{l}\mathrm{F} \\
.385\end{array}$ & $\frac{\text { Sig. }}{.681^{\mathrm{b}}}$ \\
\hline \multicolumn{2}{|c|}{$1 \quad$ Regression } & & & $\begin{array}{r}1205282373.50 \\
7\end{array}$ & \multirow[t]{3}{*}{.385} & \multirow[t]{3}{*}{$.681^{\mathrm{b}}$} \\
\hline \multicolumn{2}{|c|}{ Residual } & $\begin{array}{r}482343263303 . \\
533\end{array}$ & 154 & $\begin{array}{r}3132099112.36 \\
1\end{array}$ & & \\
\hline \multicolumn{2}{|r|}{ Total } & $\begin{array}{r}484753828050 . \\
548 \\
\end{array}$ & 156 & & & \\
\hline
\end{tabular}

a. Dependent Variable: WM_Volume

b. Predictors: (Constant), Mean_Z_Score, Age_At_Scan 

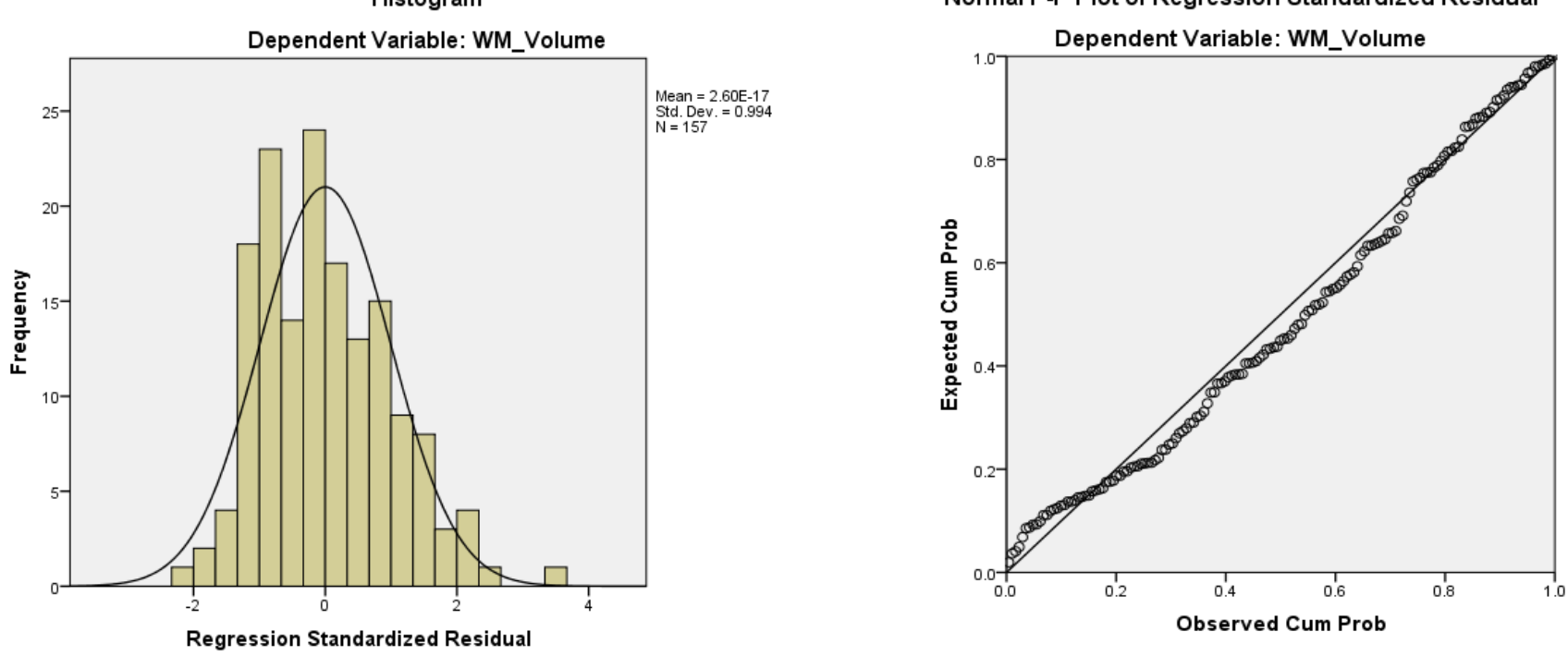

Figures 1 and 2: A histogram of the frequency by regression standardized residual in regards to the dependent variable of WM volume; expected cumulative probability by observed cumulative probability of regression of the regression standardized residual in regards to WM volume. Note a relatively high amount of standard deviation of the residual plot toward the lower end (-2) in Figure 1. Figure 2 misses a significant amount the median or mean when a linear model line was represented in the sample. The line is more skewed to the fit of the lower and higher ends of expected/observed cumulative probability.

Table 4: Non-stepwise entered coefficients of regression. The significance here is again much higher than the step-wise model at about 0.900 and 0.386 for age and mean score respectively to the constant, or dependent variable, of WM. In general, values for this type of model are much higher overall.

\begin{tabular}{|c|c|c|c|c|c|c|}
\hline \multirow[b]{2}{*}{ Model } & & \multicolumn{2}{|c|}{ Unstandardized Coefficients } & \multirow{2}{*}{$\begin{array}{c}\text { Standardized } \\
\text { Coefficients } \\
\text { Beta }\end{array}$} & \multirow[b]{2}{*}{$\mathrm{t}$} & \multirow[b]{2}{*}{ Sig. } \\
\hline & & $\mathrm{B}$ & Std. Error & & & \\
\hline \multirow[t]{3}{*}{1} & (Constant) & 569880.428 & 48468.390 & & 11.758 & .000 \\
\hline & Age_At_Scan & -422.285 & 3343.410 & -.011 & -.126 & .900 \\
\hline & $\begin{array}{l}\text { Mean_Z_Scor } \\
\text { e }\end{array}$ & -9842.634 & 11330.169 & -.073 & -.869 & .386 \\
\hline
\end{tabular}

\begin{tabular}{|r|r|r|r|r|r|r|}
\hline \multicolumn{2}{|c|}{$95.0 \%$ Confidence Interval for B } & \multicolumn{3}{|c|}{ Correlations } & \multicolumn{2}{c|}{ Collinearity Statistics } \\
\hline \multicolumn{1}{c|}{ Lower Bound } & Upper Bound & Zero-order & Partial & Part & Tolerance & \multicolumn{1}{c|}{ VIF } \\
\hline 474131.701 & 665629.154 & & & & & \\
\cline { 4 - 7 }-7027.153 & 6182.582 & .010 & -.010 & -.010 & .921 & 1.085 \\
-32225.247 & 12539.979 & -.070 & -.070 & -.070 & .921 & 1.085 \\
& & & & & & \\
\hline
\end{tabular}




\section{Standard T1-Weighted Image vs. FAST Segmented T1-Weighted Image of WM}
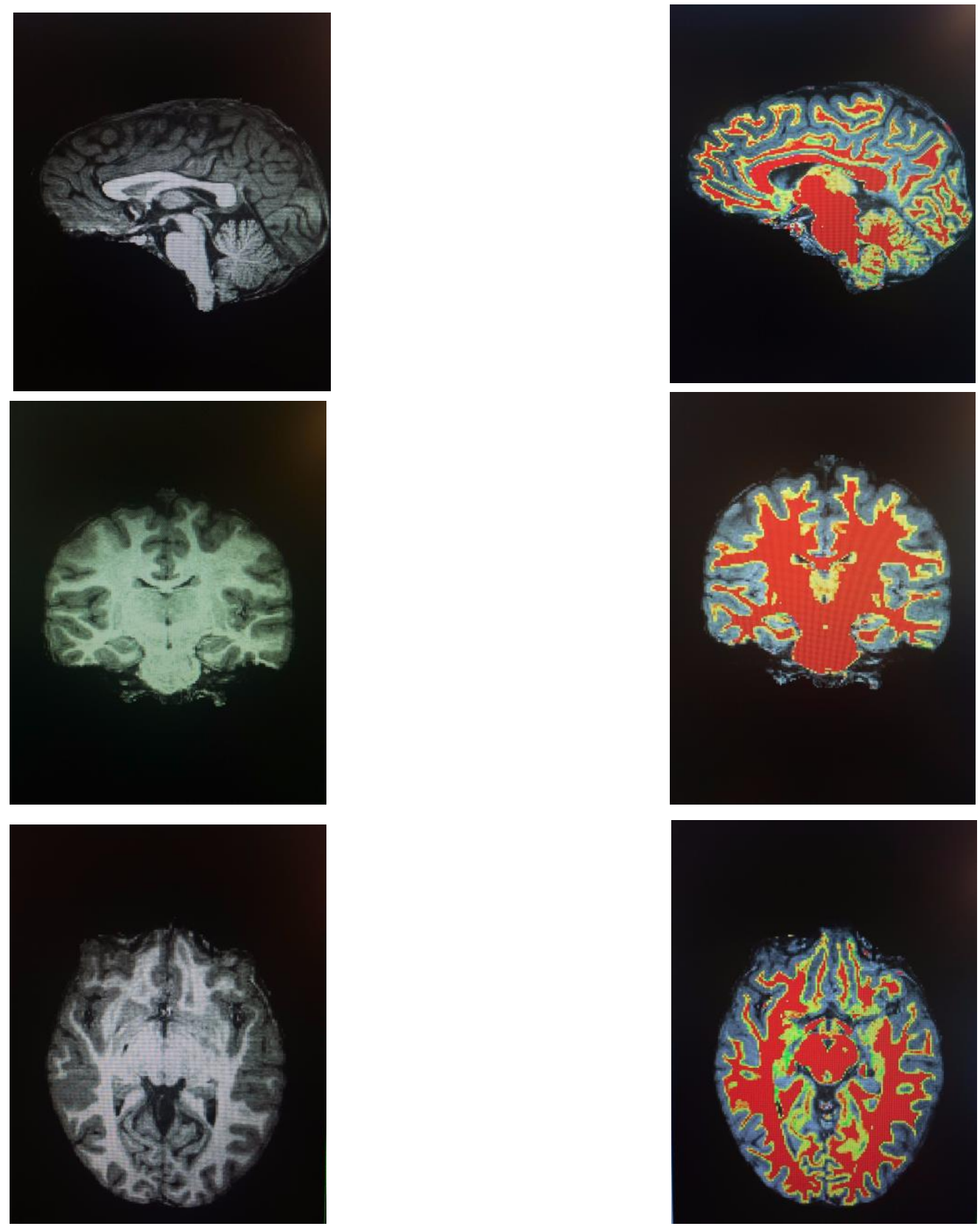

Figure 3: Examples of T1-weighted images of MRI anatomical scans used (left). The second set of images (right) demonstrates the masking performed via AFNI after the segmentation of FAST. WM. The brainstem remained in the masking quantity and FAST voxel count. Although not optimal, cortico spinal tracts report motor signals from the cerebrum to the brainstem and the spinal cord. Therefore, for the purposes of this study and identifying processing speed, the brainstem was not edited out of each T1-weighted image slice 


\section{DISCUSSION}

The purpose of this study was to determine the possible relationship between WM volume and processing speed in adolescence. As brain imaging has increased in usage within the last two decades, the present study was conducted in order to contribute to the literature of brain structure and function. Upon investigation of the sample, there was evidence to suggest that there was a positive relationship between WM volume and age, as well as a negative relationship between WM volume and age with processing speed. This was in support of the hypothesis as a negative value was used to determine a faster processing speed. Correlation and relationship values were relatively low, which may propose other variables that may need to be included when determining functionality from structure.

The present study was conducted on the basis of a large sample $(\mathrm{N}>100)$. Although there have been previous studies conducted looking at the relationship of WM and processing speed, there was a lack of investigating solely adolescents [7, 14, 24, 30,]. The original hypothesis that there would be a positive relationship between WM volume and processing speed in adolescence was marginally supported by the evidence. This might have been the result of a number of confounding variables that may have influenced the results of the study but were not taken into account.

The addition of the brainstem as a structure containing WM -- as opposed to isolating WM in the cerebral cortex and other structures -- may have affected the outcome of the analysis. WM within the brainstem may be suggested to primarily function for motor processing, albeit some regulation of cognitive processes. The present study's measures and definition for processing speed could be argued as mainly cognitive processes and not motor processing. It is suggested that a similar study be conducted to identify the role of the brainstem in cognitive processing and use the present study to complement any findings.

Sex differences for processing speed have been reported to differ between both adults and adolescents in some studies [7, 11, 13, 17]. Sex differences extend to different speeds of growth in males and females, as well as the reported difference in volumes and cranial capacities [19]. The growth rates of both WM and GM vary not only by individual but by the sex of the individual as well. The present study lacks the inclusion of sex differences in processing speed which is crucial when looking at ages of critical development; an analysis of multiple regression with additional variables such as sex - assuming these will hold with regression -- might provide further insight into the relationship between structure and function. By including additional variables, there is the possibility of higher significance within the step-wise method of regression as opposed to the limits of the two independent variables in the present study.

Adolescence is a critical period for the change in emotional and cognitive functions [33]. WM tracts and total volume of WM have been reported to grow significantly during adolescence $[13,23]$. Therefore, the present study attempted to further investigate the relationship between WM volume and processing speed. If the structure of WM was compromised either physically or developmentally, there have been cases where processing speed and cognitive functioning has been significantly affected [8]. Suggested research following this study would be further research of factors that influence changes in WM such as substance effects, lesions, and trauma. Obtaining a large sample would prove challenging for furthering this research, as well as the improbability of lesions in the developing adolescent brain. 
In conclusion, the current study did find a small relationship between WM volume and processing speed while accounting for age in a step-wise multiple regression. The current study also suggested the possible variability in processing speed scores; further investigation of large sample sizes, isolated age ranges, and many more possible variables when determining a reliable relationship between structure and function of brain regions is encouraged.

\section{ACKNOWLEDGEMENTS}

Dr. Bonnie Nagel is thanked for her generous tutelage throughout the study, as well as the provision of access to the data set in whole and direction of analysis. Scott Jones and Jesse Chiem are thanked for their extensive instruction in Linux and FAST segmentation. Kristina Hernandez is thanked for her help in processing speed determination and SPSS analysis. And I thank my family -- Jerry, Laurie, and Jason Laxamana for their unwavering support.

\section{References}

1. Asato, M. R., Terwilliger, R., Woo, J., \& Luna, B. (2010). White matter development in adolescence: a DTI study. Cerebral Cortex, 20(9), 2122-2131.

2. Assaf, Y., \& Pasternak, O. (2008). Diffusion tensor imaging (DTI)-based white matter mapping in brain research: a review. Journal of molecular neuroscience, 34(1), 51-61.

3. Bava, S., Boucquey, V., Goldenberg, D., Thayer, R. E., Ward, M., Jacobus, J., \& Tapert, S. F. (2011). Sex differences in adolescent white matter architecture. Brain research, 1375, 41-48.

4. Barnea-Goraly, N., Kwon, H., Menon, V., Eliez, S., Lotspeich, L., \& Reiss, A. L. (2004). White matter structure in autism: preliminary evidence from diffusion tensor imaging. Biological psychiatry, 55(3), 323-326.

5. Brant-Zawadzki, M., Gillan, G. D., \& Nitz, W. R. (1992). MP RAGE: a three-dimensional, T1weighted, gradient-echo sequence--initial experience in the brain. Radiology, 182(3), 769-775.

6. Bucur, Barbara, et al. "Age-related slowing of memory retrieval: contributions of perceptual speed and cerebral white matter integrity." Neurobiology of aging 29.7 (2008): 1070-1079.

7. Biswal, B. B., Mennes, M., Zuo, X. N., Gohel, S., Kelly, C., Smith, S. M., ... \& Dogonowski, A. M. (2010). Toward discovery science of human brain function. Proceedings of the National Academy of Sciences, 107(10), 4734-4739.

8. Breteler, M. M. B., Van Swieten, J. C., Bots, M. L., Grobbee, D. E., Claus, J. J., Van Den Hout, J. H. W., ... \& Hofman, A. (1994). Cerebral white matter lesions, vascular risk factors, and cognitive function in a population-based study The Rotterdam Study. Neurology, 44(7), 1246-1246.

9. Camarata, S., \& Woodcock, R. (2006). Sex differences in processing speed: Developmental effects in males and females. Intelligence, 34(3), 231-252.

10. Charlton, R. A., Barrick, T. R., McIntyre, D. J., Shen, Y., O'Sullivan, M., Howe, F. E. E. A., ... \& Markus, H. S. (2006). White matter damage on diffusion tensor imaging correlates with agerelated cognitive decline.Neurology, 66(2), 217-222. 
11. Der, G., \& Deary, I. J. (2006). Age and sex differences in reaction time in adulthood: results from the United Kingdom Health and Lifestyle Survey.Psychology and aging, 21(1), 62.

12. Davis, S. W., Dennis, N. A., Buchler, N. G., White, L. E., Madden, D. J., \& Cabeza, R. (2009). Assessing the effects of age on long white matter tracts using diffusion tensor tractography. Neuroimage, 46(2), 530-541.

13. De Bellis MD, Keshavan MS, Beers SR, Hall J, Frustaci K, Masalehdan A, et al. Sex differences in brain maturation during childhood and adolescence. Cereb Cortex. 2001;11:552-7

14. Dow, C., Seidenberg, M., \& Hermann, B. (2004). Relationship between information processing speed in temporal lobe epilepsy and white matter volume. Epilepsy \& Behavior, 5(6), 919-925.

15. Giedd JN, Snell JW, Lange N, Rajapakse JC, Casey BJ, Kozuch PL, et al. Quantitative magnetic resonance imaging of human brain development: ages 4-18. Cereb Cortex. 1996;6:551-60.

16. Gao, W., Lin, W., Chen, Y., Gerig, G., Smith, J. K., Jewells, V., \& Gilmore, J. H. (2009). Temporal and spatial development of axonal maturation and myelination of white matter in the developing brain. American journal of neuroradiology, 30(2), 290-296.

17. Gur, R. C., Turetsky, B. I., Matsui, M., Yan, M., Bilker, W., Hughett, P., \& Gur, R. E. (1999). Sex differences in brain gray and white matter in healthy young adults: correlations with cognitive performance. The Journal of Neuroscience, 19(10), 4065-4072.

18. Jernigan TL, Trauner DA, Hesselink JR, Tallal P. A Maturation of human cerebrum observed in vivo during adolescence. Brain. 1991;114 ( Pt 5):2037-49. "Magnetic Resonance, a critical peerreviewed introduction". European Magnetic Resonance Forum. Retrieved November 17, 2014.

19. Lynn, R. (1993). Further evidence for the existence of race and sex differences in cranial capacity. Social Behavior and Personality: an international journal, 21(2), 89-92.

20. Mugler, J. P., \& Brookeman, J. R. (1990). Three-dimensional magnetization-prepared rapid gradient-echo imaging (3D MP RAGE). Magnetic Resonance in Medicine, 15(1), 152-157.

21. Nagel, B. J., Medina, K. L., Yoshii, J., Schweinsburg, A. D., Moadab, I., \& Tapert, S. F. (2006). Age-related changes in prefrontal white matter volume across adolescence. Neuroreport, 17(13), 1427.

22. Oldfield, R. C. (1971). The assessment and analysis of handedness: the Edinburgh inventory. Neuropsychologia, 9(1), 97-113.

23. Olesen, P. J., Nagy, Z., Westerberg, H., \& Klingberg, T. (2003). Combined analysis of DTI and fMRI data reveals a joint maturation of white and grey matter in a fronto-parietal network. Cognitive Brain Research, 18(1), 48-57.

24. Paus, T. (2010). Growth of white matter in the adolescent brain: myelin or axon?. Brain and cognition, 72(1), 26-35

25. Penke, L., Maniega, S. M., Murray, C., Gow, A. J., Hernández, M. C. V., Clayden, J. D., ... \& Deary, I. J. (2010). A general factor of brain white matter integrity predicts information processing speed in healthy older people. The Journal of Neuroscience, 30(22), 7569-7574. 
26. Pfefferbaum A, Mathalon DH, Sullivan EV, Rawles JM, Zipursky RB, Lim KO. A quantitative magnetic resonance imaging study of changes in brain morphology from infancy to late adulthood. Arch Neurol. 1994;51:874-87

27. Reiss AL, Abrams MT, Singer HS, Ross JL, Denckla MB. Brain development, gender and IQ in children A volumetric imaging study. Brain. 1996;119 ( Pt 5):1763-74

28. Salthouse, T. A. (1996). The processing-speed theory of adult age differences in cognition. Psychological review, 103(3), 403.

29. Silveri, M. M., Tzilos, G. K., \& Yurgelun-Todd, D. A. (2008). Relationship between white matter volume and cognitive performance during adolescence: effects of age, sex and risk for drug use. Addiction, 103(9), 1509-1520.

30. Sowell, E. R., Peterson, B. S., Thompson, P. M., Welcome, S. E., Henkenius, A. L., \& Toga, A. W. (2003). Mapping cortical change across the human life span. Nature neuroscience, 6(3), 309315.

31. Tamnes, C. K., Østby, Y., Fjell, A. M., Westlye, L. T., Due-Tønnessen, P., \& Walhovd, K. B. (2010). Brain maturation in adolescence and young adulthood: regional age-related changes in cortical thickness and white matter volume and microstructure. Cerebral cortex, 20(3), 534-548.

32. Turken, U., Whitfield-Gabrieli, S., Bammer, R., Baldo, J. V., Dronkers, N. F., \& Gabrieli, J. D. (2008). Cognitive processing speed and the structure of white matter pathways: convergent evidence from normal variation and lesion studies. Neuroimage, 42(2), 1032-1044.

33. Wakana, S., Jiang, H., Nagae-Poetscher, L. M., Van Zijl, P. C., \& Mori, S. (2004). Fiber tractbased atlas of human white matter anatomy 1. Radiology,230(1), 77-87.

34. Van den Heuvel, D. M. J., Ten Dam, V. H., De Craen, A. J. M., Admiraal-Behloul, F., Olofsen, H., Bollen, E. L. E. M., ... \& Van Buchem, M. A. (2006). Increase in periventricular white matter hyperintensities parallels decline in mental processing speed in a non-demented elderly population.Journal of Neurology, Neurosurgery \& Psychiatry, 77(2), 149-153.

35. Yurgelun-Todd, D. A., Killgore, W. D., \& Young, A. D. (2002). Sex differences in cerebral tissue volume and cognitive performance during adolescence.Psychological reports, 91(3), 743-757.

36. Zhang, Y. and Brady, M. and Smith, S. Segmentation of brain MR images through a hidden Markov random field model and the expectation-maximization algorithm. IEEE Trans Med Imag, 20(1):45-57, 2001 